\title{
Structural Organization of Poly(vinyl alcohol) Hydrogels Obtained by Freezing and Thawing Techniques: A SANS Study
}

\author{
Rosa Ricciardi, ${ }^{\dagger}$, Gaetano Mangiapia, ${ }^{\dagger}$ Fabrizio Lo Celso, ${ }^{\ddagger}$ Luigi Paduano, ${ }^{\dagger}$ Roberto Triolo, \\ Finizia Auriemma, ${ }^{*}{ }^{\dagger}$ Claudio De Rosa,${ }^{\dagger}$ and Françoise Lauprêtre ${ }^{\S}$ \\ Dipartimento di Chimica, Complesso Universitario di M.S. Angelo, Università degli Studi di Napoli \\ "Federico II", Via Cinthia, 80126 Napoli (NA), Italy, Dipartimento di Chimica Fisica, Viale delle Scienze, \\ Università degli Studi di Palermo, Parco d'Orleans II, 90128 Palermo (PA), Italy, and Laboratoire de \\ Recherche sur les Polymères, UMR 7581-CNRS, 2 à 8 rue Henri Dunant, 94320 Thiais, France
}

Received August 17, 2004. Revised Manuscript Received December 10, 2004

\begin{abstract}
The structural organization of matter in poly(vinyl alcohol) (PVA) hydrogels obtained by repeatedly freezing and thawing dilute solutions of PVA in $\mathrm{D}_{2} \mathrm{O}$ is investigated by use of small-angle neutron scattering measurements (SANS). This study is the first systematic and quantitative investigation in the medium range of length scales on PVA hydrogels obtained by freezing and thawing techniques. The studied gels have a complex hierarchical structure, extending over a wide range of length scales. The structural organization on the micron length scale originates from the presence of two separated phases constituted by polymer-rich and polymer-poor regions. The network structure may be interpreted in terms of the connectivity of the regions occupied by the polymer-rich phase, which extend over the macroscopic dimensions of the sample. The organization on the medium length scale is provided by the presence of small crystallites, fringed micelle-like, within the polymer-rich phase. In these regions, the crystals are highly connected by swollen amorphous tie chains. The presence of these tie chains ensures the connectivity of the macroscopic network. The structural organization on the short length scale is essentially provided by the relative arrangement of chains within the crystallites and in the swollen amorphous zones. The PVA hydrogel structure has been modeled as a collection of polydisperse and homogeneous spherical crystallites, interacting via hard-spheres potential. SANS experiments permitted us to obtain values of the crystallite size of about $33 \AA$, of the volume fraction of polymer-rich phase of the order of $1 \%$ and a value of the average crystallite-crystallite correlation distances of the order of a few tens of nanometers, independent of the imposed number of freeze/thaw cycles $(n)$, for $n>1$. The present analysis also indicates that the structure of the gel obtained imposing a single freeze/thaw cycle is somehow intermediate between the structure of the homogeneous starting solution and the structure of the already well-formed sample obtained by imposing two consecutive cycles.
\end{abstract}

\section{Introduction}

Atactic poly(vinyl alcohol) (PVA) is the largest volume, water-soluble synthetic polymer manufactured in the United States and in Japan. ${ }^{1}$ It has vast applications in packaging, textiles, and pervaporation membranes. The main characteristics of PVA are (i) its semicrystalline character despite its lack of stereoregularity ${ }^{2-4}$ and (ii) a strong tendency to exhibit both inter- and intramolecular hydrogen bonds.

Thermoreversible hydrogels ${ }^{5,6}$ of PVA, obtained from dilute PVA water solutions by repeatedly freezing at -20 ${ }^{\circ} \mathrm{C}$ and then thawing at room temperature, show a number

* To whom correspondence should be addressed. Phone: +39-081674341. Fax: +39-081674090. E-mail: auriemma@chemistry.unina.it.

† Università degli Studi di Napoli "Federico II".

¥ Università degli Studi di Palermo.

\& Laboratoire de Recherche sur les Polymères, UMR 7581-CNRS.

(1) Finch, C. A. Polyvinyl Alcohol: Developments; Wiley: Chichester, U.K., 1992.

(2) Bunn, C. W.; Peiser, H. S. Nature 1947, 159, 161-162.

(3) Bunn, C. W. Nature 1948, 161, 929-930.

(4) Sakurada, I.; Fuchino, K.; Okada, N. Bull. Inst. Chem. Res. Kyoto Univ. 1950, 23, 78.

(5) Yokoyama, F.; Masada, I.; Shimamura, K.; Ikawa, T.; Monobe, K. Colloid Polym. Sci. 1986, 264, 595-601. of interesting properties. They have good mechanical properties and high water content, and they are stable at room temperature and able to retain their original shape. In addition, they are biocompatible. All these properties make PVA hydrogels suitable for different biomedical and pharmaceutical applications. ${ }^{7-9}$ In particular, their high mechanical strength and high elasticity are a few of the attributes of this material that make it suitable for applications as artificial tissues and contact lenses. ${ }^{8,10,11}$

From the pioneering works of Pines and Prins ${ }^{12}$ it was proposed that spinodal liquid-liquid-phase separation, followed by crystallization of parts of the PVA chains, is the origin of gel formation in PVA dilute solution in water.

(6) Guenet, J. M. Thermoreversible Gelation of Polymers and Biopolymers; Academic Press: San Diego, CA, 1992.

(7) Peppas, N. A.; Mongia, N. K. Eur. J. Pharm. Biopharm. 1997, 43, $51-58$.

(8) Hassan, C. M.; Peppas, N. A. Adv. Polym. Sci. 2000, 153, 37-65.

(9) Lozinsky, V. I. Russ. Chem. Rev. 1998, 67, 573-586.

(10) Hyon, S.-H.; Cha, W.-I.; Ikada, Y.; Kita, M.; Ogura, Y.; Honda, Y. J. Biomater. Sci., Polym. Ed. 1994, 5, 397-406.

(11) Tamura, K.; Ike, O.; Hitomi, S.; Isobe, J.; Shimizu, Y.; Nambu, M. Trans.-Am. Soc. Artif. Intern. Organs 1986, 32, 605-609.

(12) Pines, E.; Prins, W. Macromolecules 1973, 6, 888-895. 
Komatsu et al. ${ }^{13}$ investigated the relationship between physical gelation and liquid-liquid-phase separation (spinodal decomposition) of PVA aqueous solutions, over a wide range of PVA concentration and temperatures, and they obtained a phase diagram. They found that spinodal decomposition is not necessary for gelation but has a significant effect on gelation at temperatures lower than $0{ }^{\circ} \mathrm{C}$ for PVA aqueous solutions with PVA content higher than $5 \%$ w/w; moreover, in agreement with Pines and Prins, gelation starts by hydrogen bonding followed by crystallization. ${ }^{14,15}$ The role of liquid-liquid-phase separation in the physical gelation of PVA aqueous solutions was also investigated by $\mathrm{Wu}$ et al., 16,17 who described the structure of aqueous PVA gels in terms of a two-phase model: highly interconnected polymerrich domains which meander in regions of a polymerdepleted phase. The size of the phase-separated domains is of the order of microns, thus accounting for the opaque aspect of PVA hydrogels. The macroscopic network structure may be interpreted in terms of connectivity of polymer-rich regions.

It is now accepted that the freeze/thaw PVA gelation process results in the formation of a porous network in which polymer crystallites act as junction points. $5,8,9,13,18-29$ The outstanding physical properties of freeze/thaw PVA hydrogels, indeed, strongly depend on the crystallinity of the samples. They also depend on their hierarchical structure, which extends over a very wide spatial scale. ${ }^{9,19,20,30}$ The organization on the large length scale (of the order of microns) originates from the presence of two separated phases constituted by polymer-rich and polymer-poor regions. In agreement with studies by Pines and Prins, ${ }^{12}$ the network structure may be interpreted in terms of the connectivity of the regions occupied by the polymer-rich phase, which extend over the macroscopic dimensions of the sample. The organization on the medium length scales is

(13) Komatsu, M.; Inoue, T.; Miyasaka, K. J. Polym. Sci., Part B: Polym. Phys. 1986, 24, 303-311.

(14) Morawetz, H. Macromolecules in Solution; Interscience Publisher: New York, 1965.

(15) te Nijenhuis, K. Adv. Polym. Sci. 1997, 130, 177.

(16) Wu, W.; Shibayama, M.; Roy, S.; Kurokawa, H.; Coyne, L. D.; Nomura, S.; Stein, R. S. Macromolecules 1990, 23, 2245-2251.

(17) Wu, W.; Kurokawa, H.; Roy, S.; Stein, R. S. Macromolecules 1991, $24,4328-4333$

(18) Peppas, N. A. Die Makromol. Chem. 1975, 176, 3433-3440.

(19) Watase, M.; Nishinari, K. J. Polym. Sci., Part B: Polym. Phys. 1985, $23,1803-1811$

(20) Watase, M.; Nishinari, K. Makromol. Chem. 1989, 190, 155-163.

(21) Willcox, P. J.; Howie, D. W., Jr.; Schimdt-Rohr, K.; Hoagland, A.; Gido, S. P.; Pudjijanto, S.; Kleiner, L. W.; Venkatraman, S. J. Polym. Sci., Part B: Polym. Phys. 1999, 37, 3438-3454.

(22) Ricciardi, R.; Gaillet, C.; Ducouret, G.; Lafuma, F.; Lauprêtre, F. Polymer 2003, 44, 3375-3380.

(23) Ricciardi, R.; Auriemma, F.; De Rosa, C.; Lauprêtre, F. Macromolecules 2004, 37, 1921-1927.

(24) Kanaya, T.; Ohkura, M.; Kaji, H.; Furusaka, M.; Misawa, M. Macromolecules 1994, 27, 5609-5615.

(25) Takeshita, H.; Kanaya, T.; Nishida, K.; Kaji, K. Macromolecules 1999, $32,7815-7819$

(26) Kanaya, T.; Ohkura, M.; Takeshita, H.; Kaji, H.; Furusaka, M.; Yamaoka, H.; Wignall, G. D. Macromolecules 1995, 28, 3168-3174

(27) Kanaya, T.; Takeshita, H.; Nishikoji, Y.; Ohkura, M.; Nishida, K.; Kaji, K. Supramol. Sci. 1998, 5, 215-221.

(28) Takeshita, H.; Kanaya, T.; Nishida, K.; Kaji, K.; Takahashi, T.; Hashimoto, M. Phys. Rev. E 2000, 61, 2125-2128.

(29) Takeshita, H.; Kanaya, T.; Nishida, K.; Kaji, K. Physica B 2002, 311 , $78-83$.

(30) Hassan, C. M.; Peppas, N. A. Macromolecules 2000, 33, 2472-2479. provided by the presence of small crystallites, fringed micelle-like, within the polymer-rich phase. In these regions, the crystals are highly connected by tie chains. The presence of these tie chains, which constitute a sort of swollen amorphous region, ensures the connectivity of the macroscopic network. The structural organization at short range is described by the relative arrangement of chains in the fringed micelle-like crystals and by the interactions between close neighboring chain segments in the swollen amorphous region.

Quantitative information concerning the structural organization of PVA gels formed in mixtures of DMSO and water on various length scales have been obtained by Kanaya et al. using wide- ${ }^{24,27}$ and small-24,26,27,29 angle neutron scattering and light-scattering ${ }^{25-27}$ techniques. These studies confirmed that cross-linking in these gels is ensured by small crystallites. Besides, the crystallites were shown to have a sharp surface; the average size of the crystallites $(\sim 70 \AA)$ and the average distance between crystallites (of the order of 150-200 $\AA$ ) in their gels were also estimated. Moreover, these gels possess a supra-organization due to the presence of domain boundaries between polymer-rich and polymer-poor phases, the size of these regions being of the order of $1-2 \mu \mathrm{m} . .^{25-29}$

In the short range of length scale, the structural organization of freeze/thaw PVA hydrogels has been studied by X-ray diffraction. ${ }^{5,19-21}$ These experiments support the hypothesis that PVA hydrogels have a porous structure, with pores mainly occupied by water. ${ }^{23}$ The X-ray diffraction profiles of these gels were interpreted as arising from the sum of three contributions due to the scattering of "free" water, crystalline aggregates, and amorphous PVA swollen by the solvent. In agreement with measurements performed with other techniques, ${ }^{21-23,31}$ it was observed that the degree of crystallinity and the size of crystallites increase with increasing the number of freeze/thaw cycles.

In the present paper, we will investigate PVA hydrogels, obtained at different numbers of freeze/thaw cycles, and provide detailed structural information in the medium range of mesoscopic length scales, by using small-angle neutron scattering measurements (SANS). The range of scattering vector, $q=4 \pi \sin \theta / \lambda$, (where $2 \theta$ is the scattering angle) used in SANS measurements $\left(2 \times 10^{-3} \AA^{-1}\right.$ to $\left.0.12 \AA^{-1}\right)$ permits us to obtain information concerning the structure of hydrogels in the range of a few tens of nanometers.

\section{Experimental Section}

Materials. The gel samples analyzed in the present work were obtained by following the same protocol used by some of us in previous papers. ${ }^{22,23,31}$ They were prepared by using an atactic PVA sample (Aldrich ref 36,315-4) with an average molecular weight, $M_{w}$, of 115500 and a degree of hydrolysis of $98-99 \%$. The ${ }^{13} \mathrm{C}$ NMR spectrum analysis of PVA in deuterated water solution showed that the percentages of $m m, m r$, and $r r$ configurational triads are $22.1,50.1$, and $27.8 \%$, respectively, confirming that the polymer is atactic.

PVA aqueous solutions (11\% w/w) were prepared by dissolving the PVA polymer in deuterated water (Aldrich, stated purity $99.8 \%$,

(31) Ricciardi, R.; Auriemma, F.; Gaillet, C.; De Rosa, C.; Lauprêtre, F. Macromolecules 2004, 37, 9510-9516. 
molar mass $20.03 \mathrm{~g} \mathrm{~mol}^{-1}$ ) at $96{ }^{\circ} \mathrm{C}$, under reflux and stirring, for about $3 \mathrm{~h}$. The polymer was entirely dissolved and the obtained homogeneous solution was slowly cooled to room temperature and kept at this temperature for one night to eliminate air bubbles.

The aqueous PVA solutions were then poured between glass slides with 1-mm spacers, at room temperature. We checked that the solutions did not jellify and remained transparent when left at room temperature in a sealed tube for more than one month.

Strong physical PVA hydrogel films were obtained by subjecting the polymer aqueous solutions to several repeated freeze/thaw cycles, consisting of a 20 -h freezing step at $-22^{\circ} \mathrm{C}$ followed by a 4-h thawing step at $25^{\circ} \mathrm{C}$. The as-formed PVA hydrogels obtained by 1 to 9 freeze/thaw cycles are denoted as samples GEL-1 to GEL-9.

PVA hydrogels undergo syneresis upon application of repeated freeze/thaw cycles, expelling some water on their surface. For this reason, the polymer weight concentration of each gel was determined by weighting each sample in the swollen and in the corresponding dried state. The polymer weight fractions of GEL- $n$ samples are reported in Table 1. They are higher than the polymer weight fraction of the starting solution $(11 \% \mathrm{w} / \mathrm{w})$ and increase with increasing the number of freeze/thaw cycles.

PVA crystalline films were obtained by drying as-prepared GEL- $n$ samples in air at room temperature.

X-ray Measurements. Wide-angle X-ray powder diffraction profiles were collected at room temperature with a Philips diffractometer using $\mathrm{Ni}$-filtered $\mathrm{Cu} \mathrm{K} \alpha$ radiation $(\lambda=1.5418 \AA)$ and scans at $0.005 \operatorname{deg}(2 \theta) / \mathrm{s}$ in the $2 \theta$ range from 10 to $60^{\circ}$. To prevent the sample from drying during the experiment, the diffraction profiles were recorded using a homemade brass sample holder placed in a special brass chamber covered with an out-of-focus Mylar film, in an atmosphere saturated with vapors of the mother solution. During the time needed for recording the diffraction patterns $(\sim 3 \mathrm{~h})$, the weight loss of the sample was less than 2 wt \%

Apparent crystalline dimensions along the [101] lattice direction were calculated by measuring the width at half-height of the corresponding Bragg reflection and applying the Scherrer formula ${ }^{32}$

$$
t=\frac{k \cdot \lambda}{\beta \cdot \cos \theta}
$$

where $t$ is the apparent crystalline dimension along a given lattice direction, $k$ is a constant ( $k=0.89 \mathrm{rad}), \lambda$ is the wavelength of the $\mathrm{X}$-rays, $\beta$ is the width at half-height (expressed in radians), and $\theta$ is the Bragg angle. Due to the low intensity of the Bragg peak at $2 \theta=19.4^{\circ}$ in the crystalline PVA hydrogels, the standard deviation associated with the so-determined apparent crystalline dimensions is of the order of $3 \AA$.

SANS Measurements. Small-angle neutron scattering measurements were performed at the KWS2 facility located at the Forschungszentrum of Jülich, Germany. Samples were contained in 1-mm path-length quartz cells in order to prevent the drying of gels. Measurement times ranged between 20 min and $8 \mathrm{~h}$. Neutrons with an average wavelength $\lambda$ of $7 \AA$ and a wavelength spread $\Delta \lambda / \lambda \leq 0.2$ were used. A two-dimensional array detector at three different sample-to-detector distances, 2, 8, and $20 \mathrm{~m}$, detected neutrons scattered from the samples. These configurations permitted collection of the scattered intensity in a range of scattering vectors between 0.002 and $0.12 \AA^{-1}$.

Raw data were corrected for electronic background and empty cell scattering. Detector sensitivity corrections and transformation

(32) Klug, H. P.; Alexander, L. E. X-ray Diffraction Procedures; John Wiley: New York, 1959; p 512.

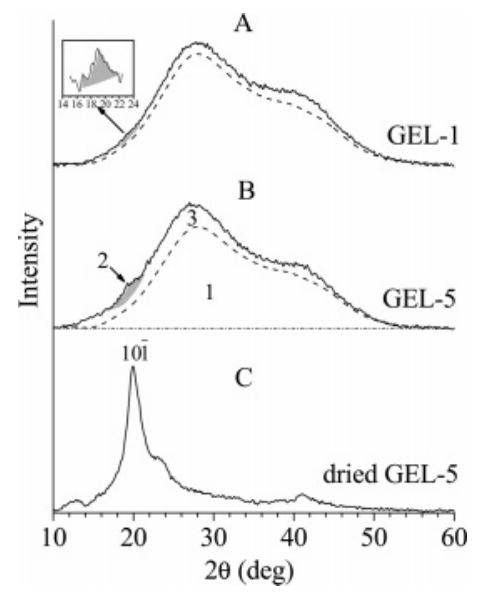

Figure 1. X-ray powder diffraction profiles of freshly prepared PVA hydrogel samples (A and B) and of dried gel (C), obtained after 1 (A) and 5 freeze/thaw cycles (B and $\mathrm{C}$, continuous lines). The $\mathrm{X}$-ray diffraction profile of liquid $\mathrm{D}_{2} \mathrm{O}$ is also reported (dashed line). The crystalline reflection apparent in the X-ray diffraction profiles of gels in $2 \theta$ range $16-22^{\circ}$ is evidenced in gray; it corresponds to the $10 \overline{1}$ reflection of the crystalline PVA, indicated in $\mathrm{C}$. The inset in part A shows the X-ray diffraction intensity in the $2 \theta$ range $14-24^{\circ}$, on enlarged scales for GEL- 1 .

to absolute scattering cross-sections $(\mathrm{d} \sigma / \mathrm{d} \Omega)(q)$ were made with a secondary Lupolene standard according to the equation

$$
\frac{\mathrm{d} \sigma}{\mathrm{d} \Omega}(q)=\left(\frac{\mathrm{d} \sigma}{\mathrm{d} \Omega}\right)_{\mathrm{L}}=\frac{d_{\mathrm{L}} T_{\mathrm{L}} L_{\mathrm{S}}^{2}}{d_{\mathrm{S}} T_{\mathrm{S}} L_{\mathrm{L}}^{2} I_{\mathrm{L}}}\left[\left(I_{\mathrm{S}}-I_{\mathrm{bck}}\right)-T_{\mathrm{S}}\left(I_{\mathrm{EC}}-I_{\mathrm{bck}}\right)\right]
$$

where the subscripts $\mathrm{L}$ and $\mathrm{S}$ are for Lupolene and sample, respectively. $d$ and $T$ are the cell thickness and transmission, respectively, whereas $L_{\mathrm{S}}$ and $L_{\mathrm{L}}$ are detector distances at which sample and Lupolene were measured to obtain the scattering intensities $I_{\mathrm{S}}$ and $I_{\mathrm{L}}$. Raw data were also corrected for intensity of background $I_{\mathrm{bck}}$ and intensity of empty cell $I_{\mathrm{EC}}$. Data were then radially averaged and absolute scattering cross-sections were obtained.

\section{Experimental Results}

The X-ray diffraction profile of as-prepared GEL-1 and GEL-5 hydrogel samples along with the X-ray diffraction profile of the dried GEL-5 sample are reported in Figure 1 as an example, after subtraction of a straight baseline which approximates the background contribution. For comparison, the X-ray diffraction pattern of pure deuterated water, which is the major component of these gels, is also indicated in Figure $1 \mathrm{~A}$ and $\mathrm{B}$ (dashed line). The diffraction profiles of the gels (Figure 1A and B) exhibit two halos centered at $2 \theta \approx 28$ and $41^{\circ}$, as in the diffraction profile of pure water, and a weak peak in the $2 \theta$ range $16-22^{\circ}$ which corresponds to the $10 \overline{1}$ reflection of crystalline PVA (Figure $1 \mathrm{C}$ ). This result demonstrates the presence of a low amount of small crystalline PVA aggregates in the gel sample. The intensity of this reflection drastically increases going from GEL-1 to GEL-5, indicating that the amount of crystalline phase increases.

With reference to Figure $1 \mathrm{~B}$ and consistent with the analysis performed in a previous paper, ${ }^{23}$ the X-ray diffraction profile of GEL- $n$ samples is considered as the sum of three contributions: a large contribution (area A1) due to the scattering of pure $\mathrm{D}_{2} \mathrm{O}$ (dashed curves), a small diffraction 
Table 1. Polymer Concentration, Total $\mathrm{D}_{2} \mathrm{O}$ Content, Fractional Amount of Free $\mathrm{D}_{2} \mathrm{O}$, Degree of Crystallinity $\left(x_{c}\right)$, Fraction of Swollen Amorphous PVA, Fraction of Crystalline PVA with Respect to the Sum of the Crystalline Fraction and the Swollen Amorphous Component $\left(f_{c}\right)$, and Apparent Crystallite Dimensions (along the [101] Lattice Direction) of Freshly Prepared PVA/D $\mathbf{D}_{2} \mathrm{O}$ Gels Obtained by Different Numbers of Freeze/Thaw Cycles

\begin{tabular}{|c|c|c|c|c|c|c|c|}
\hline gel sample & $\begin{array}{l}\text { polymer } \\
\text { concn } \\
(\% \mathrm{w} / \mathrm{w})^{a}\end{array}$ & $\begin{array}{l}\text { total } \mathrm{D}_{2} \mathrm{O} \\
(\% \mathrm{w} / \mathrm{w})^{a}\end{array}$ & $\begin{array}{c}\text { free } \mathrm{D}_{2} \mathrm{O} \\
(\%)^{b}\end{array}$ & $\begin{array}{c}x_{c} \\
(\%)^{b}\end{array}$ & $\begin{array}{l}\text { swollen } \\
\text { amorphous } \\
(\%)^{b}\end{array}$ & $\begin{array}{c}f_{c} \\
(\%)^{b}\end{array}$ & $\begin{array}{l}\text { apparent dimensions } \\
\text { of crystallites } \\
(\AA)^{b}\end{array}$ \\
\hline GEL-1 & 12.0 & 88.0 & 86.2 & 0.4 & 13.4 & 2.5 & 28 \\
\hline GEL-3 & 12.7 & 87.3 & 84.2 & 0.8 & 15.0 & 4.8 & 34 \\
\hline GEL-5 & 13.4 & 86.6 & 80.9 & 1.1 & 18.0 & 5.6 & 35 \\
\hline GEL-7 & 14.7 & 85.3 & 80.1 & 1.1 & 18.8 & 5.7 & 38 \\
\hline GEL-8 & 13.7 & 86.3 & 78.5 & 1.4 & 20.2 & 6.3 & 40 \\
\hline GEL-9 & 14.9 & 85.1 & 78.1 & 1.4 & 20.6 & 6.3 & 39 \\
\hline
\end{tabular}

${ }^{a}$ Determined by gravimetric measurements. ${ }^{b}$ Determined by X-ray powder diffraction experiments.

component in the range from 16 to $22^{\circ}$ due to the crystalline aggregates (area A2), and a third component (area A3) due to the presence of amorphous PVA swollen by water molecules.

As suggested by some of us, ${ }^{23}$ we have measured the areas $\mathrm{A} 1, \mathrm{~A} 2$, and $\mathrm{A} 3$ of the three components and then determined the relative amount of "free $\mathrm{D}_{2} \mathrm{O}$ " in the gels through the ratio $\mathrm{A} 1 /(\mathrm{A} 1+\mathrm{A} 2+\mathrm{A} 3)$. The degree of crystallinity $\left(x_{c}\right)$ and the fraction of the swollen amorphous PVA phase have been approximately evaluated by the ratios $\mathrm{A} 2 /(\mathrm{A} 1+\mathrm{A} 2$ $+\mathrm{A} 3)$ and $\mathrm{A} 3 /(\mathrm{A} 1+\mathrm{A} 2+\mathrm{A} 3)$, respectively. Finally, the relative amount of crystalline PVA with respect to the sum of the crystalline and swollen amorphous portions, $f_{c}$ (=A2/ $(\mathrm{A} 2+\mathrm{A} 3))$ has been also determined. The values thus obtained and the apparent crystalline dimensions along the [101] lattice direction, determined using the Scherrer formula, are reported in Table 1.

In agreement with the results of ref 23 , from the data reported in Table 1 it is apparent that the polymer concentration in as-formed PVA hydrogels slightly increases with increasing the number of freeze/thaw cycles, whereas the relative amount of "free water" decreases. These latter values are only slightly lower than the values of the effective weight fraction of $\mathrm{D}_{2} \mathrm{O}$ determined by gravimetric measurements. The degree of crystallinity increases with increasing the number $n$ of freeze/thaw cycles, and the values of the relative amount of crystalline PVA with respect to the sum of the crystalline and swollen amorphous portions, $f_{c}$, determined in the present analysis, are in agreement with those determined using different techniques on similar systems. ${ }^{21-23,31}$ Moreover, the apparent crystalline dimensions (along the [10י1] lattice direction) increase with $n$. It is worth noting that, going from GEL-1 to GEL-9, the degree of crystallinity $\left(x_{\mathrm{c}}\right)$ increases from 0.4 to $1.4 \%$, whereas the polymer concentration increases only from $\sim 12$ to $\sim 15 \%$ w/w. Therefore, as suggested in ref 23 , the increase of crystallinity with $n$ is not only the result of an increase of polymer concentration in the gel, but is also due to the significant improvement of the gel structure as a consequence of freeze/ thaw cycles; in fact, each new cycle not only improves the structure of the water-swollen PVA chains connecting the crystals, but also induces an increase of the size and, to a lesser extent, of the number of the crystallites. ${ }^{21-23,31}$

SANS spectra obtained for fresh PVA gel samples after $1,2,3,5$, and 9 cycles are reported in Figure $2 \mathrm{~A}-\mathrm{E}, \mathrm{A}^{\prime}$, and $\mathrm{E}^{\prime}$. The SANS profile of the $\mathrm{PVA} / \mathrm{D}_{2} \mathrm{O}(11 \% \mathrm{w} / \mathrm{w})$ starting solution used for gel preparation is shown in Figure
$2 \mathrm{~A}$ and $\mathrm{A}^{\prime}$. The scattering cross-section profiles $(\mathrm{d} \sigma / \mathrm{d} \Omega)$ of GEL- $n$ samples present a background higher than the profile of the mother solution; moreover, the background scattering of GEL- $n$ samples increases with increasing $n$. This increase is fully accounted for by the different polymer concentrations of the various samples. The polymer concentrations of GEL- $n$ samples, indeed, increase with increasing the number of freeze/thaw cycles, and, for all gels, are higher than the polymer concentration of the mother solution (see Table 1).

Inspection of Figure $2 \mathrm{~A}-\mathrm{E}$ leads to some qualitative considerations. First of all, the scattering cross-section profile of the homogeneous starting solution appears quite different from those of PVA GEL- $n$ samples, confirming that, in the latter systems, PVA chains and solvent molecules are highly organized. In the SANS profiles of the GEL- $n$ samples we can distinguish the following three different regions. (1) A region at low $q$ values $\left(q<0.009 \AA^{-1}\right)$. In this region the scattering cross section exhibits an upturn, which is clearly not present in the scattering cross-section curve of the homogeneous starting solution. In this zone, the data could reflect a supramolecular organization, which we associate with the presence of two separated phases constituted by polymer-rich and polymer-poor regions. (2) A region at intermediate $q$ values $\left(0.009<q<0.035 \AA^{-1}\right)$. In this region an inflection point is present, which gives an indication of the average distance between the scattering crystallites (given approximately by $2 \pi / q$ ). In all gel samples this inflection point is at $q \approx 0.03 \AA^{-1}$, indicating a distance between crystallites of the order of $200 \AA$. (3) A region where 0.035 $<q<0.08 \AA^{-1}$. In this zone, scattering cross-sections decrease with a power law $\mathrm{d} \sigma / \mathrm{d} \Omega(q) \propto q^{-D}$, with the value of $D$ depending on the number of cycles. More precisely $D \cong 2$ for GEL-1, $D \cong 3$ for GEL-2, GEL-3, and GEL-9, while $D=3.5$ for GEL-5. Provided we are looking in this region at the boundary structure between two phases, possibly the crystallites and the swollen amorphous region in the polymer-rich phase, we may apply the surface fractal concept to the function $\mathrm{d} \sigma / \mathrm{d} \Omega(q)$. According to this concept, the exponent $D$ is related to the surface fractal dimension $d_{s}$ in a $d$-dimensional space, through $D=2 d-d_{s}$. For example, in 3-dimensional space, $d_{s}$ ranges from 2 to 3 , corresponding to a range of $D$ from 4 to 3 . If the boundary were smooth, Porod's law $\left(\mathrm{d} \sigma / \mathrm{d} \Omega(q) \propto q^{-4}\right)$ would be observed. For our PVA gels, values of $D$ less than 4 suggest that the boundary is not very clear, or it would not be a boundary in the case of GEL-1. 

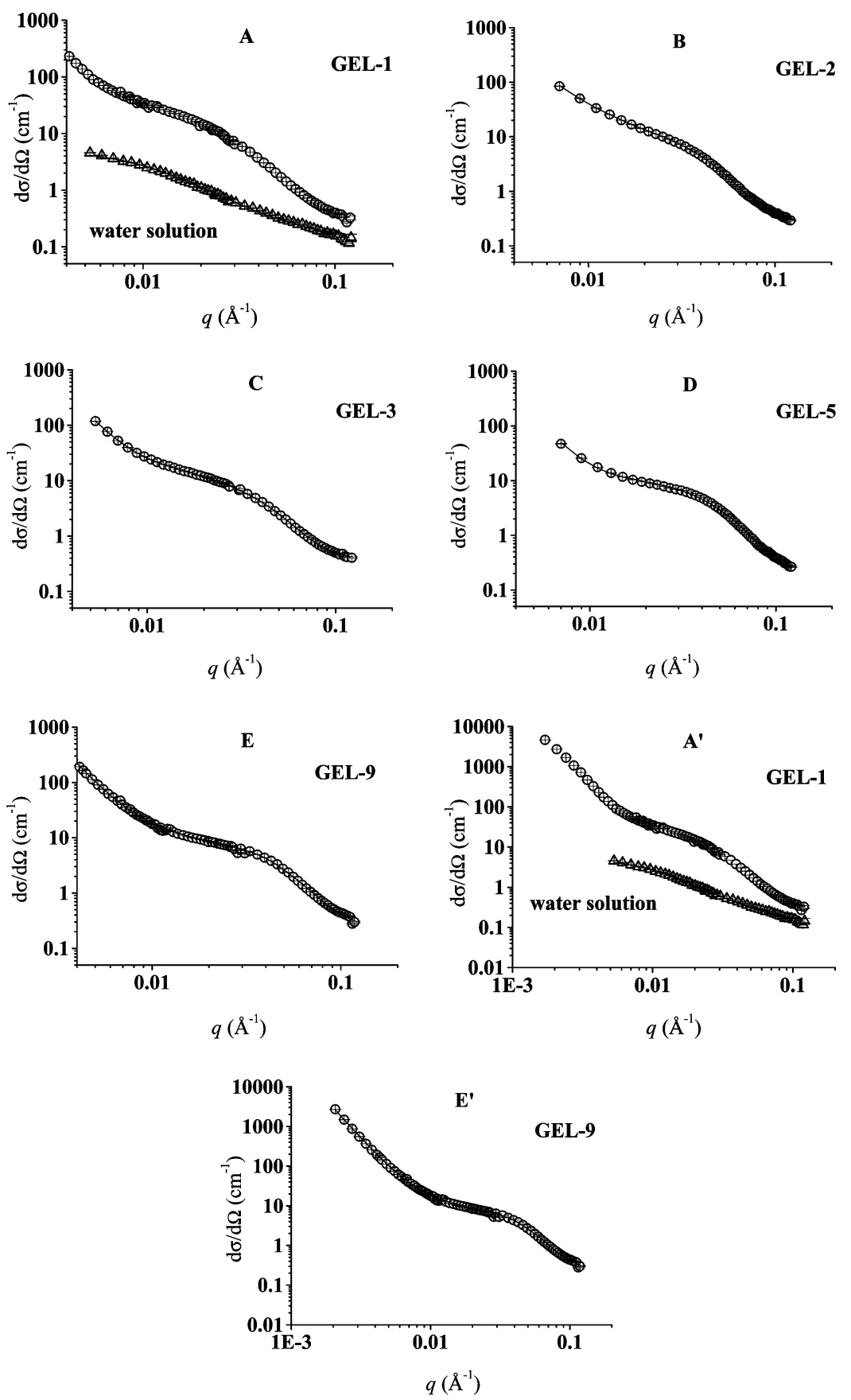

Figure 2. SANS data for as-prepared GEL- $n(\mathrm{O})$ and starting PVA solution $(11 \% \mathrm{w} / \mathrm{w}, \triangle)$ : GEL-1 and starting PVA solution (A and A'), GEL-2 (B), GEL-3 (C), GEL-5 (D), GEL-9 (E and E'). Solid lines are fitting curves obtained by using eq 4 for GEL- $n$ and by using eq 3 for starting PVA solution. For GEL-1 and GEL-9 samples data collected in a wider $q$ range, $2 \times 10^{-3}$ to $0.12 \AA^{-1}$, are also reported ( $\mathrm{A}^{\prime}$ and $\mathrm{E}^{\prime}$ ).

Data Analysis. To have quantitative information on the collected scattering data, we have set up a least-squares fitting procedure. Models used in the fits depend on the analyzed systems. Therefore, in the following, we report the expressions used for PVA homogeneous solution and for PVA hydrogels.

PVA Solution. SANS data obtained for the PVA starting solution indicate the presence of small scattering objects, essentially the individual chains of polymer with a Gaussian coil conformation. According to the Debye model, ${ }^{33}$ the scattering cross-section for a collection of molecules in such

(33) Debye, P. J. Phys. Colloid Chem. 1947, 51, 18-32. conformation is given by the equation

$$
\frac{\mathrm{d} \sigma}{\mathrm{d} \Omega}(q)=2 c L_{A}\left(\rho_{\mathrm{PVA}}-\rho_{0}\right)^{2} V^{2} \frac{q^{2} \bar{R}_{g}^{2}-1+\exp \left(-q^{2} \overline{R_{g}}{ }^{2}\right)}{q^{4}{\overline{R_{g}}}^{4}}+
$$

where $c$ is the molar concentration of PVA, $L_{A}$ is the Avogadro constant, $\overline{R_{g}}$ is the average gyration radius of the polymer, $V$ is the volume of a PVA molecule, $\rho_{\mathrm{PVA}}$ and $\rho_{0}$ are the scattering length densities of PVA $\left(\rho_{\mathrm{PVA}}=7.5 \times\right.$ $\left.10^{-7} \AA^{-2}\right)$ and $\mathrm{D}_{2} \mathrm{O}\left(\rho_{0}=6.34 \times 10^{-6} \AA^{-2}\right)$, respectively, and $(\mathrm{d} \sigma / \mathrm{d} \Omega)_{\text {inc }}$ is the incoherent scattering cross section. 
Table 2. Values of $k_{f r}$ (see eq 4), Average Radius of Crystallites $(\bar{R})$, Hard-Sphere Interaction Radius $\left(R_{\mathrm{HS}}\right)$, Fractal Dimension $m$ (see eq 4$)$, Crystallite Volume Fraction in Gels $(\phi)$, and Polydispersion Index of Crystallites Radii $\left(I_{p}\right)$ for As-Formed GEL-n (with $\left.n=2,3,5,9\right)$, Obtained by Fitting SANS Data with Eq 4

\begin{tabular}{ccccccc}
\hline sample & $k_{f r}\left(\mathrm{~cm}^{-1} \AA^{-m}\right)$ & $\bar{R}(\AA)$ & $R_{H S}(\AA)$ & $m$ & $\phi$ & $I_{p}$ \\
\hline GEL-2 & $(4.58 \pm 0.21) 10^{-5}$ & $33.6 \pm 0.9$ & $230 \pm 5$ & $2.73 \pm 0.08$ & 0.013 & $1.15 \pm 0.02$ \\
GEL-3 & $(1.92 \pm 0.3) 10^{-6}$ & $31.4 \pm 0.8$ & $234 \pm 6$ & $3.27 \pm 0.1$ & 0.0076 & $1.14 \pm 0.02$ \\
GEL-5 & $(1.21 \pm 0.4) 10^{-7}$ & $35.3 \pm 0.8$ & $274 \pm 8$ & $3.78 \pm 0.1$ & 0.012 & $1.07 \pm 0.02$ \\
GEL-9 & $(8.9 \pm 0.8) 10^{-7}$ & $34.8 \pm 0.7$ & $224 \pm 5$ & $3.39 \pm 0.1$ & 0.0093 & $1.09 \pm 0.02$
\end{tabular}

For the $(11 \% \mathrm{w} / \mathrm{w}) \mathrm{PVA}-\mathrm{D}_{2} \mathrm{O}$ solution, the $\overline{R_{g}}$ value, obtained by fitting eq 3 to the experimental data, is $158 \pm 7 \AA$. This value is in good agreement with the value of $163 \AA$ determined by Nagy et al. by size exclusion chromatography for PVA solutions in $\mathrm{D}_{2} \mathrm{O}$ with concentrations ranging from 0.25 to $0.50 \%$ (w/w). The degree of hydrolysis of the PVA sample used by the authors was of $98 \%$ and its molecular weight $M_{w}$ was $110000 . .^{34}$

PVA Hydrogels. The PVA hydrogel structure has been modeled as a collection of polydisperse and homogeneous spherical crystallites, interacting via hard-spheres potential. Although crystallites are interconnected with amorphous chains, we have neglected the scattering of these amorphous chains, ${ }^{26}$ treating it as a flat background added to the incoherent scattering cross section.

The theoretical expression used for such a system is given by the following equation: ${ }^{35}$

$$
\begin{array}{r}
\frac{\mathrm{d} \sigma}{\mathrm{d} \Omega}(q)=N_{b}\left(\rho_{P V A}-\rho_{0}\right)^{2}\left\langle|F(q)|^{2}\right\rangle\left[1+\frac{|\langle F(q)\rangle|^{2}}{\left\langle|F(q)|^{2}\right\rangle}(S(q)-\right. \\
1)]+k_{f r} q^{-m}+\left(\frac{\mathrm{d} \sigma}{\mathrm{d} \Omega}\right)_{\text {inc }}
\end{array}
$$

where $N_{b}$ is the density number of the crystallites, $\rho_{\mathrm{PVA}}$ and $\rho_{0}$ are the scattering length densities of the PVA crystallites $\left(\rho_{\text {PVA }}=7.5 \cdot 10^{-7} \times \AA^{-2}\right)$ and of the solvent, $\mathrm{D}_{2} \mathrm{O}\left(\rho_{0}=6.34\right.$ $\times 10^{-6} \AA^{-2}$ ), respectively, $\left\langle|F(q)|^{2}\right\rangle$ and $S(q)$ are the form and structure factors of the scattering crystallites, $(\mathrm{d} \sigma / \mathrm{d} \Omega)_{\text {inc }}$ is the incoherent scattering cross section, and $k_{f r} q^{-m}$ is a term related to the fractal nature of the network at low $q$ values. The form factor contains information on the shape of the scattering objects, while the structure factor $S(q)$ accounts for interparticle correlations and is normally observed for concentrated or charged systems. In eq 4 the form factor is averaged for the presence of polydisperse bodies.

For homogeneous spheres $F(q)$ is given by ${ }^{36}$

$$
F(q)=\frac{4}{3} \pi R^{3} \frac{3 j_{1}(q R)}{q R}
$$

where $R$ is the radius of spheres and $j_{1}(x)$ is the first-order Bessel function:

$$
j_{1}(x)=\frac{\sin (x)-x \cos (x)}{x^{2}}
$$

(34) Nagy, D. J. Am. Lab. (Shelton, Conn.) 2003, 35, 38-43.

(35) Kotlarchyk, M.; Chen, S. H. J. Chem. Phys. 1983, 79, 2461-2469.

(36) Hayter, J. B.; Penfold, J. J. Chem. Soc., Faraday Trans. 1 1981, 77, $1851-1863$

(37) Zimm, B. H. J. Chem. Phys. 1948, 16, 1099-1116.

(38) Schultz, G. V. Z. Phys. Chem., Abt. (B) 1939, 43, 25-46.

(39) Percus, J. K.; Yevick, G. J. Phys. Rev. 1952, 110, 1-13.
Writing in explicit form $\left\langle|F(q)|^{2}\right\rangle$ and $|\langle F(q)\rangle|^{2}$ we obtain

$$
\begin{aligned}
\left\langle|F(q)|^{2}\right\rangle & =\int_{0}^{\infty}|F(q)|^{2} f(R) d R \\
|\langle F(q)\rangle|^{2} & =\left|\int_{0}^{\infty}\right| F(q)|f(R) d R|^{2}
\end{aligned}
$$

where $f(R)$ is the distribution function of the radii.

A Zimm-Schultz distribution has been assumed for the radii of spheres ${ }^{37,38}$

$$
f(R)=\left(\frac{Z+1}{\bar{R}}\right)^{Z+1} \frac{R^{Z}}{\Gamma(Z+1)} \exp \left(-\frac{Z+1}{\bar{R}} R\right)
$$

In eq $9, \bar{R}$ is the average radius of the spheres, $Z$ is a parameter depending on the width of the distribution, and $\Gamma$ is the Euler Gamma function. Using eqs 5 and 9, it is possible to evaluate integrals 7 and 8 analytically. ${ }^{35}$

As already mentioned, the structure factor used to analyze SANS data takes into account the hard-sphere repulsions between the crystallites. An analytical form for $S(q)$ is obtainable in the Percus - Yevick approximation. ${ }^{39}$ It is given by ${ }^{40}$

$$
S(q)=\frac{1}{1+24 \phi \frac{G\left(q R_{\mathrm{HS}}, \phi\right)}{q R_{\mathrm{HS}}}}
$$

where $\phi$ is the volume fraction of the bodies, $R_{\mathrm{HS}}$ is the hardsphere interaction radius, and $G(y, \phi)$ (with $y=q R_{\mathrm{HS}}$ ) is a function given by the following:

$$
\begin{aligned}
G(y, \phi) & =\frac{(1+2 \phi)^{2}}{(1-\phi)^{4} y^{2}}(\sin y-y \cos y)- \\
& \frac{6 \phi(1+\phi / 2)^{2}}{(1-\phi)^{4} y^{3}}\left[2 y \sin y+\left(2-y^{2}\right) \cos y-2\right]+ \\
& \frac{\phi(1+2 \phi)^{2}}{2(1-\phi)^{4} y^{5}}\left\{-y^{4} \cos y+4\left[\left(3 y^{2}-6\right) \cos y+\right.\right. \\
& \left.\left.\left(y^{3}-6 y\right) \sin y+6\right]\right\}
\end{aligned}
$$

Therefore, by inserting eqs 5-11 into eq 4, we obtain an expression for the scattering cross section which depends on $N_{b}, k_{f r}, \bar{R}, R_{\mathrm{HS}}, m, Z$, and $(\mathrm{d} \sigma / \mathrm{d} \Omega$ ) inc. The volume fraction $\phi$ is correlated to the other parameters through the equation

$$
\phi=\frac{4}{3} \pi \bar{R}^{3} N_{b}
$$

The values of the above-mentioned parameters have been optimized using a least-squares procedure to fit eq 4 to the experimental SANS data of Figure 2. Table 2 reports the

(40) Magid, L. J.; Triolo, R.; Johnson, J. S., Jr. J. Phys. Chem. 1984, 88, $5730-5734$ 


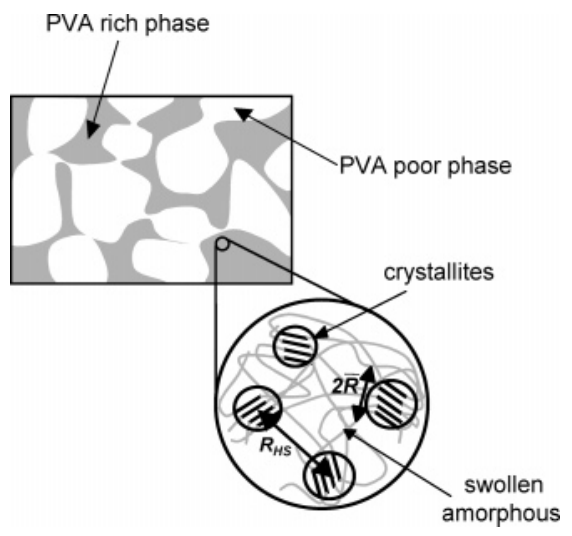

Figure 3. Bicontinuous structure of PVA hydrogels obtained by freeze/ thaw cycles with a PVA-rich phase and a PVA-poor phase. Circles in the insert represent, schematically, the hard-spheres model used for fitting SANS equations to the experimental data.

obtained results in the case of GEL- $n$ samples for $n=2,3$, 5 , and 9; in this table the polydispersion index $I_{p}$ replaces the $Z$ parameter. The relationship between $I_{p}$ and $Z$ is given by the following equation:

$$
I_{p}=\frac{\int_{0}^{\infty} f(R) R^{2} d R}{\left(\int_{0}^{\infty} f(R) R d R\right)^{2}}=\frac{Z+2}{Z+1}
$$

The best fits for the experimental SANS data of GEL- $n$ samples for $n=2,3,5$, and 9 are indicated in Figure 2B-E and $\mathrm{E}^{\prime}$ (solid lines).

Within the present framework, where the SANS intensity is dominated by the crystallite-crystallite interference, the $\phi$ value roughly corresponds to the volume fraction of crystallites in the gel. Moreover, the hard-sphere interaction radius, $R_{\mathrm{HS}}$, corresponds to the average distance between the crystallites whereas $\bar{R}$ may be taken as a measure of the average size of crystallites, as illustrated in Figure 3, where a model of the bicontinuous structure of PVA hydrogels is shown.

Results given in Table 2 clearly show that, for GEL- $n$ samples with $n$ higher than 1 , the values of $R_{\mathrm{HS}}$ are of the order of $250 \AA$. The size of crystallites remains constant $(\sim 33 \AA)$ and is close to the values determined by X-ray diffraction (see Table 1). The polydispersion index, $I_{p}$, is in all cases close to 1.1, indicating a narrow distribution of the crystallite sizes. Finally, the $\phi$ values are close to $1 \%$ and appear in fair agreement with the corresponding values of crystallinity $\left(x_{c}\right)$ determined by X-ray diffraction.

The case of GEL-1 sample deserves a separate discussion. The SANS data of this gel could not be interpreted in terms of reasonable values of fitting parameters, using the present model, probably because the small fraction of crystallites present in this gel have a smooth surface and cannot be modeled in terms of a collection of polydisperse and homogeneous spherical crystallites, interacting via hardspheres potential, where the scattering of amorphous chains connecting the crystallites as tie-chains can be neglected. This fact is consistent with other observations concerning the structure of GEL-1 samples. In fact, PVA hydrogels obtained by imposing a single freeze-thaw cycle are less crystalline (Table 1 and Figure 1) and exhibit mechanical properties poorer than those of other gels, showing low values of mechanical strength and shear elastic modulus. ${ }^{5,21-23,41,42}$

The present analysis indicates that the main differences between GEL- 1 and GEL- $n$ samples with $n$ higher than 1 is probably due to the fact that the gel structure of GEL-1 is not yet well defined. Although the structure of the freeze/ thaw PVA hydrogels is imprinted already during the first freeze/thaw cycle, ${ }^{8,21,23}$ the structure of GEL-1 may be considered somehow intermediate between the structure of the homogeneous starting solution and the structure of the already well-formed GEL-2 sample, in the sense that GEL-1 results from only partial segregation phenomena (i.e., incomplete phase separation and formation of only few and small crystallites). During the first freeze/thaw cycle, indeed, the homogeneous solution undergoes a phase separation leading to the formation of a polymer-rich and a polymer-poor phase, accompanied by crystallization of PVA in the polymer-rich phase. ${ }^{12,13,21,27,28}$ Since the freezing procedure is quite fast, the phase separation occurring during the first cycle may be not complete and only repeating the freeze-thaw procedure leads to gels with a well-defined network scaffolding.

\section{Conclusions}

The present study is the first systematic and quantitative investigation in the medium range of length scale on PVA hydrogels obtained by freezing and thawing techniques. The range of $q$ values sampled in the SANS experiments $(2 \times$ $10^{-3}$ to $1.2 \times 10^{-1} \AA^{-1}$ ) has allowed us to access information concerning the structure of hydrogels in terms of concentration fluctuations involving correlation lengths of the order of tens of nanometers. The SANS data obtained for the PVA gels have been interpreted in terms of a model consisting of a collection of polydisperse and homogeneous spherical crystallites interacting via a hard-spheres potential. Within this framework, the values of crystallite size (of the order of $33 \AA$ ) and polymer volume fraction of crystals (of the order of $1 \%$ ), obtained by fitting the SANS experimental curves to calculated diffraction profiles, are in good agreement with corresponding values determined by using other techniques. In addition, SANS experiments permitted evaluation of an average crystallite-crystallite correlation distance, $R_{\mathrm{HS}}$, of the order of $250 \AA$ for GEL- $n$ samples with $n$ higher than 1 . The present analysis also indicates that the structure of GEL-1 is somehow intermediate between the structure of the homogeneous starting solution and the structure of the already well-formed GEL-2 sample.

Acknowledgment. The Centro di Competenza "Nuove Tecnologie per le Attività Produttive" Regione Campania P.O.R. 2000-2006 Misura 3.16 is gratefully acknoweledged for the financial support. We thank the Institut für Festkörperforschung of Jülich for provision of beam time.

\section{CM048632Y}

(41) Ricciardi, R.; D’Errico, G.; Auriemma, F.; Ducouret, G.; Tedeschi, A.; De Rosa, C.; Lauprêtre, F.; Lafuma, F. Chem. Mater. submitted for publication.

(42) Urushizaki, F.; Yamaguchi, H.; Nakamura, K.; Numajiri, S.; Sugibayashi, K.; Morimoto, Y. Int. J. Pharm. 1990, 58, 135-142. 